\title{
Types of urethral catheters for management of short-term voiding problems in hospitalized adults: a short version Cochrane Review
}

\author{
K Schumm ${ }^{1}$, T B L Lam \\ Sri Lanka Journal of Obstetrics and Gynaecology 2009; 31: 110-121 \\ Cochrane Database of Systematic Reviews 2008, Issue 2. Art. No: CD004013. DOI: 10.1002/14651858.CD004013.pub3. \\ Copyright (C) 2008 The Cochrane Collaboration. Published by John Wiley E Sons, Ltd. \\ This article is reproduced with the kind permission of the authors and the publisher.
}

\begin{abstract}
Background: Urinary tract infection (UTI) is the most common hospital acquired infection. The major associated cause is indwelling urinary catheters. Currently there are many types of catheters available. A variety of specialized urethral catheters have been designed to reduce the risk of infection. These include antiseptic impregnated catheters and antibiotic impregnated catheters. Other issues that should be considered when choosing a catheter are ease of use, comfort and cost.

Objectives: The primary objective of this review was to determine the effect of type of indwelling urethral catheter on the risk of urinary tract infection in adults who undergo short-term urinary catheterization.

Methods: We searched the Specialized Trials Register of the Cochrane Incontinence Group (searched September 11, 2007). We also examined the bibliographies of relevant articles and contacted catheter manufacturer representatives for trials. All randomized and quasi-randomized trials comparing types of indwelling urinary catheters for short-term catheterization in hospitalized adults. Shortterm catheterization was defined as up to and including 14 days, or other temporary short-
\end{abstract}

This paper is based on a Cochrane review published in The Cochrane Library 2008, Issue 2 (see www. thechochranelibrary.com for information). Cochrane Reviews are regularly updated as new evidence emerges and in response to feedback, and The Cochrane Library should be consulted for the most recent version of the review. If you wish to comment on this or other Cochrane Reviews, please use the Cochrane Library Feedback System. The results of a Cochrane Review can be interpreted differently, depending on people's perspectives and circumstances. Please consider the conclusions presented carefully. They are the opinions of the review authors, and are not necessarily shared by The Cochrane Collaboration.

${ }^{1}$ Trial Manager, Academic Urology Unit/CHaRT, University of Aberdeen, Health Sciences Building, Foresterhill, Aberdeen AB25 2ZB, UK. Corresponding author: K Schumm, E-mail: k.schumm@abdn.ac.uk term use as defined by the trialists (for example $<21$ days with data time points at 7-day intervals). Data were extracted by one reviewer and independently verified by a second reviewer for both the original review and for the update. Disagreements were resolved by discussion. Data were processed as described in the Cochrane Handbook. Where data in trials were not fully reported, clarification was sought directly from the trialists (secondary sources were used to confirm results of one trial).

Results: Twentythree trials met the inclusion criteria involving 5,236 hospitalized adults in 22 parallel group trials and 27,878 adults in one large cluster-randomized cross-over trial. The antiseptic catheters were either impregnated with silver oxide or silver alloy. Silver oxide catheters were not associated with a statistically significant reduction in bacteriuria in short-term catheterized hospitalized adults but the confidence intervals were wide (RR 0.89, 95\% CI 0.68-1.15) and these catheters are no longer available. Silver alloy catheters were found to significantly reduce the incidence of asymptomatic bacteriuria (RR 0.54, 95\% CI 0.43-0.67) in hospitalized adults catheterized for $<1$ week. At $>1$ week of catheterization the risk of asymptomatic bacteriuria was still reduced with the use of silver alloy catheters (RR 0.64, 95\% CI 0.51$0.80)$. The randomized cross-over trial of silver alloy catheters versus standard catheters was excluded from the pooled results because data were not available prior to crossover. The results of this trial indicated benefit from the silver alloy catheters and included an economic analysis that indicated cost savings of between $3.3 \%$ and $35.5 \%$. Antibiotic impregnated catheters were compared to standard catheters and found to lower the rate of asymptomatic bacteriuria in the antibiotic group at $<1$ week of catheterization for both minocycline and rifampicin combined (RR 0.36, 95\% CI 0.180.73 ), and nitrofurazone (RR $0.52,95 \%$ CI $0.34-$ $0.78)$. However, at $>1$ week the results were not statistically significant. One of 56 men in the 
antibiotic impregnated group had a symptomatic UTI compared with 6 of 68 who had standard catheters (RR 0.20, 95\% CI $0.03-$ 1.63). Three trials compared two different types of standard catheters (defined as catheters that are not impregnated with antiseptics or antibiotics) to investigate infection. Individual trials were too small to show whether or not one type of standard catheter reduced the risk of catheter related urinary tract infection compared to another type of standard catheter.

Conclusions: The results suggest that the use of silver alloy indwelling catheters for catheterizing hospitalized adults short-term reduces the risk of catheter acquired urinary tract infection. Further economic evaluation is required to confirm that the reduction of infection compensates for the increased cost of silver alloy catheters. Catheters impregnated with antibiotics are also beneficial in reducing bacteriuria in hospitalized adults catheterized for $<1$ week but the data were too few to draw conclusions about those catheterized for longer. There was not enough evidence to suggest whether or not any standard catheter was better than another in terms of reducing the risk of urinary tract infection in hospitalized adults catheterized short term. Siliconized catheters may be less likely to cause urethral side effects in men; however, this result should be interpreted with some caution as the trials were small and the outcome definitions and specific catheters compared varied.

Neurourol. Urodynam. 27:738-746, 2008. ${ }^{\circ} 2008$ Wiley-Liss, Inc.

Key words: UTI; urethral, catheter; short-term

\section{Background}

Urinary tract infection (UTI) is the most common hospitalacquired infection. A major associated cause is indwelling urinary catheters ${ }^{1-4}$. These infections can be serious and lead to significant morbidity and increase in length of hospital stay. In the United States catheter-associated urinary tract infections make up $40 \%$ of all hospital-acquired infections with the proportion of these connected or contributing to mortality assessed as approximately $3 \%{ }^{4}$.

The risk of developing bacteriuria from a catheter increases by between $3 \%$ and $6 \%$ per day that a catheter is in situ. This result in approximately $50 \%$ of hospitalized patients catheterized for longer than 7-10 days contracting an infection ${ }^{4}$. Factors that also increase the risk of infection include female gender, older age and co-morbidities. ${ }^{4}$

The criterion for diagnosis of UTI in a noncatheterized population is generally agreed to be a positive urine culture (bacteriuria $>105 \mathrm{CFU} / \mathrm{ml}$ ), with symptomatic UTI being diagnosed using the same criteria plus associated symptoms. Catheter associated UTI is diagnosed at a lower level of bacteriuria $(>102$ or $>103 \mathrm{CFU} / \mathrm{ml}$ ) for asymptomatic infections, plus associated symptoms for symptomatic diagnosis. Unless antimicrobial drug therapy is commenced the bacteriuria will naturally progress to concentrations of $>105$ usually within $72 \mathrm{hr} .5$ Symptomatic bacteriuria is characterized by the presence of dysuria, urgency, frequency and hematuria. Further symptoms indicative of pyelonephritis such as fever and flank pain develop in $<1 \%$ of patients with catheter associated bacteriuria.4 For purposes of this review, a positive urine culture in the absence of symptoms is defined as asymptomatic bacteriuria rather than asymptomatic UTI.

The individual microbial species responsible for catheter associated UTI vary widely between hospitals and within hospitals ${ }^{4,6}$. Escherichia coli remains the most common pathogen causing hospital-acquired UTIs, but other major species are Enterococci, Pseudomonas aeruginosa, Candida spp. and Klebsiella pneumoniae ${ }^{7}$. Generally, infection is introduced via two routes after catheterization: the intraluminal route via the inside lumen of the catheter, or the trans-urethral route where the micro-organisms enter along the surface of a sheath of mucus between the catheter and the urethra ${ }^{4}$.

Currently, there are many types of catheters available. Standard indwelling catheters are made from a variety of materials including: polyvinyl chlorine, plastic, plain latex, polytetrafluoroethylene (PTFE), silicone elastomer, pure silicone hydrogel, and polymer hydromer ${ }^{8,9}$. Specialized urethral catheters have been developed specifically to reduce the risk of infection. A common approach is to coat the catheter with anti-microbial agents either on the outer surface, the lumen, or both ${ }^{10}$. These agents may be antiseptic or antibiotic. The most common impregnation agent is silver. Silver ions are bactericidal, have been found topically to be non-toxic to humans, and have been used successfully in other areas such as controlling infection in wounds ${ }^{4}$. It is unclear whether antimicrobial resistance to silver may develop ${ }^{11}$. A previous review suggested that silver alloy catheters were more effective than silver oxide in preventing infection ${ }^{10}$. Since that review was published, there have been more trials of silver-coated catheters. Antibiotic-impregnated catheters have also been 
developed using various different types of antibiotic as the active component.

The aim of our review was to investigate the effects of the more widely used types of urethral catheters in reducing the incidence of urinary tract infection for short-term catheterization in hospitalized patients, but also taking into account other factors, such as ease of use, comfort and cost that may influence the choice. For the purpose of this review, short-term was defined as up to and including 14 days or other temporary short-term use as defined by the trialists.

\section{Objectives}

The primary objective of this review was to determine the effect of type of indwelling urethral catheter on the risk of urinary tract infection in hospitalized adults who undergo short-term catheterization.

Secondary objectives included:

- identification of the most cost-effective catheter in terms of cost per infection avoided;

- identification of the catheter that provides the most comfort to patients;

- identification of any adverse effects related to catheter use other than urinary tract infection.

We wish to make the following comparisons:

1. Antiseptic impregnated indwelling urethral catheters versus standard indwelling urethral catheters.

2. Antibiotic impregnated indwelling urethral catheters versus standard indwelling urethral catheters.

3. Antibiotic impregnated indwelling urethral catheters versus antiseptic impregnated indwelling urethral catheters.

4. One type of antiseptic impregnated indwelling urethral catheter versus another type of antiseptic impregnated indwelling urethral catheter.

5. One type of antibiotic impregnated indwelling urethral catheter versus another type of antibiotic impregnated indwelling urethral catheter.

6. One type of standard indwelling urethral catheter versus another type of standard indwelling urethral catheter.

\section{Methods}

See full version of Cochrane Review ${ }^{12}$.

\section{Description of studies}

\section{Included studies}

Thirty-five possibly eligible studies were identified, five of which were identified during the review update ${ }^{13-17}$. Twelve were excluded. The reasons for exclusion are listed in the table of Characteristics of Excluded Studies (see full version of Cochrane Review).

Twenty-three studies were included. Of these studies three trials were published in restricted format: one as a letter to the editor ${ }^{18}$ and two as abstracts from scientific meetings ${ }^{15,19}$. None of these trialists were contactable, therefore additional information on the trials came from secondary sources ${ }^{10,20}$. Furthermore, one of the trials was an unpublished report ${ }^{16}$. All other trials were published as full text articles.

\section{Design}

Twenty-two of the included studies were parallel group randomized controlled trials ${ }^{13,15-19,21-34}$ and one was a cluster-randomized cross-over trial ${ }^{35}$ comparing two or three types of indwelling urinary catheters. The 22 parallel group randomized controlled trials included 5,236 hospitalized adults (mean $n=208$, range $n=27-1,309)$. The clusterrandomized cross-over trial was a large study including 27, 878 hospitalized adults.

\section{Participants}

The trials involved heterogeneous population groups.

- Several trials ${ }^{13,19,21,23,25,26,28-30,32,34,35}$ included both women and men catheterized for hemodynamic monitoring or post-operative drainage for a variety of diagnoses.

- Three trials $22,24,33$ included men with urological diagnoses.

- Two other trials involved men only, one involved men postcardiac surgery ${ }^{27}$ and the other included men with a variety of medical and surgical diagnoses ${ }^{31}$.

- There was one trial which did not describe the characteristics of the population ${ }^{15}$.

- The distribution of men and women was not even between groups in six trials $14,17,25,26,29,34$. In one trial the intervention group (silver oxide catheter) had nearly twice the number of women than the comparison group ${ }^{29}$. The Lee trial included fewer women in the treatment group than the control group and more men in the treatment group than the control. 
- Five trials reported the number of participants on systemic antibiotics ${ }^{13,17,23,29,32}$. The numbers were similar across the groups in the trials. Four trials reported on those taking systemic antibiotics prior to catheterization ${ }^{13,17,29,32}$ and one on the number commenced on antibiotics for the final $48 \mathrm{hr}$ of catheterization $^{23}$.

\section{Interventions and Comparisons}

The majority of trials randomized participants to an antiseptic impregnated catheter or a standard catheter: however, three different types of antiseptics were investigated: silver oxide, silver alloy, and silver alloy hydrogel. One trial25 randomized participants into three arms: silver alloy, standard (hydrogel coated), and standard (non-coated). Five trials investigated antibiotic impregnated catheters versus standard catheters. Five other trials randomized participants to two different types of standard catheters.

\section{Duration of catheterization}

There was variation in the duration of catheterization in the trials. The trials described the length of catheterization in the following ways: total catheterization time, total mean length of catheterization for all participants, and mean length of catheterization in the intervention and control groups. In one trial the participants were catheterized for $48 \mathrm{hr},{ }^{27}$ while another three trials had a total length of catheterization of 5-6 days s $^{25,26,33}$ and two further trials had a total catheterization time of 14 days $^{22,34}$. Three trials recorded the length of catheterization as total mean duration catheterized for all participants. The mean time catheterized varied from $44.9 \mathrm{hr}$ in one trial $^{28}$ and 2.2, 3, and 5 days in the other three.16,31,34 Seven trials described the length of catheterization as a separate mean for intervention and control groups. One trial reported a mean length of catheterization of 3 days in the intervention group and 2 days in the control group ${ }^{17}$. The mean length of catheterization in both groups in four of the trials ranged from 3.4 to 4.6 days, ${ }^{14,21,23,29}$ while one trial had a mean length of catheterization in the intervention group of 7.7 days and 7.5 in the control group, ${ }^{30}$ and another of 7.9 in the intervention group and 7.2 in the control group ${ }^{13}$. Four trials did not clearly specify the length of catheterization $^{15,18,19,35}$.

\section{Outcome measures}

The definition of asymptomatic bacteriuria ranged from $>10^{2} \mathrm{CFU} / \mathrm{ml}$ to greater than $106 \mathrm{CFU} /$ $\mathrm{ml}$, while symptomatic bacteriuria was defined by the trialist as being "diagnosed by the healthcare provider" ${ }^{\prime 22}$. Urethritis was defined as penile discharge and/or penile discomfort in one trial ${ }^{27}$, and measurement via scanning electron microscopic analysis was used to indicate urethral inflammatory reaction in another trial ${ }^{31}$. The measurement and exact definition for catheter-related pain was not described in the only trial to investigate this outcome ${ }^{30}$. Takeuchi also separated for urethral discharge and allergic reaction (not defined).

Outcomes of interest that were included in the trials of standard catheters were catheter-related infection; however in addition one small Japanese trial $^{30}$ reported data for: catheter-associated pain, urethral discharge, and catheterrelated hypersensitivity or allergy. The five trials that randomized participants to two different types of standard catheters ${ }^{21,27,28,31,33}$ investigated outcomes of catheterrelated infection, catheter-related urethritis, and urethral inflammatory reaction.

\section{Primary outcome}

Apart from variation in the definition of infection, the timing of the outcome measurement and duration of followup was also diverse. Only six trials ${ }^{16,17,22,23,26,28}$ monitored catheter care violations. The method of obtaining urine specimens was varied. The majority of trials acquired samples from the catheter sampling port $\mathrm{t}^{17-19,22,23,26,30}$. However, two trials used suprapubic puncture to obtain urine samples from participants ${ }^{34}$, and one trial took specimens from the catheter urine bag, which could have resulted in contamination ${ }^{25}$.

\section{Other outcomes (including economic)}

The timing of outcome measurement of the three trials ${ }^{27,30,31}$ that investigated outcomes related to comfort and/or urethritis differed considerably. The Nacey trial investigated urethritis with assessment at 8 weeks and 6 months post-catheterization (length of catheterization 2 days) by clinical examination and urethral swabs. The method for gathering the data on catheter-related pain, urethral discharge and allergic reaction was not described in the Takeuchi trial report. The Talja trial investigated inflammatory reaction in the urethra assessed by cytological urethral swabs taken immediately after catheterization, after removal of the catheter and on the second or third day after removal. An economic analysis was included in only one trial ${ }^{35}$. The analysis included an estimation of both a lower and higher approximation of costs.

\section{Methodological quality of included studies}

Full details of the trials and an evaluation of their methodological quality are given in the full version of the Cochrane Review. 


\section{Results}

\section{Antiseptic catheter versus standard catheter}

There were two types of antiseptic catheters compared with a standard catheter: silver oxide and silver alloy. The trials for each were analyzed in two subgroups depending on the type of intervention catheter and separated into separate outcomes dependent upon duration of catheterization.

\section{Silver oxide}

Three trials compared silver oxide coated catheters with a standard catheter ${ }^{23,29,30}$. The trials used different standard catheters as the comparison catheter. Johnson used an all silicone catheter as the standard catheter, while Riley used silicone-coated latex as the comparison catheter. Takeuchi did not define the standard catheter used in the trial. Eighty percent of participants in one of the trials received systemic antibiotics (the reasons were not stated in the trial $)^{29}$. All trials included men and women admitted to surgical and/or medical wards, although Riley had more women in the treatment group (451/ $745)$ than in the control group $(285 / 564)$. In the Takeuchi trial all the participants were bacteriuria when the trial ended at 9 days catheterization.

Pooling the results of all three trials using a fixed effects model did not provide enough evidence to show whether or not there was a reduction in risk of developing bacteriuria (RR 0.89, 95\% CI 0.68-1.15, Comparison 01.01.01, Fig. 1). There was no statistically significant difference in the number with bacteriuria between groups. Subgroup analysis was possible for the Riley trial only.

\section{Subgroup analysis}

Subgroup analysis was possible in one trial ${ }^{29}$. For bacteriuria reported separately in women andmen, there was a reduction of risk of almost a third with the silver oxide catheter for women (RR 0.63 , 95\% CI 0.450.89 ), while for men there was not enough evidence to suggest whether or not there was a difference in risk with the standard catheter (RR 1.62, 95\% CI 0.91-2.88). The trial reported separately on those participants commenced on antibiotics prior to catheterization but did not state the reason for the antibiotics. Further subgroup analysis of all participants receiving systemic antibiotics indicated that combining antibiotics with silver oxide catheters may reduce the risk of bacteriuria (RR 0.67 95\% CI 0.45-0.99). Further analysis of women andmen separately who received systemic antibiotics suggested that women were protected from bacteriuria with silver oxide catheters (RR 0.50, 95\% CI 0.31-0.79), but there was not enough evidence either way for men (RR 1.02, 95\% CI 0.492.13).

\section{Silver alloy}

Nine trials compared silver alloy catheters with a standard catheter15,18,19,25,26,32,34,35. Most trials included both men and women except for one which included only men after radical prostatectomy ${ }^{34}$ and two trials

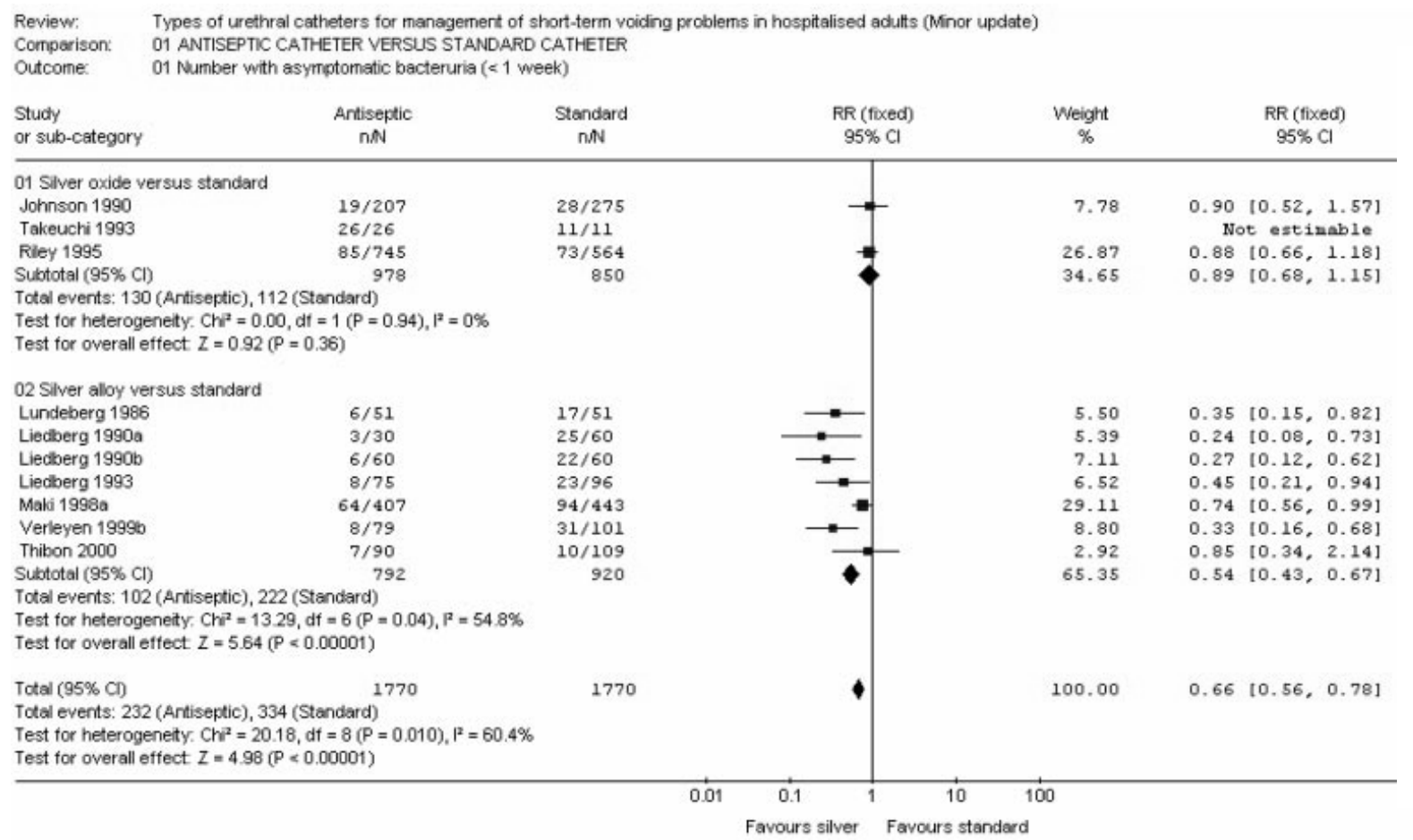

Figure 1. Antiseptic catheter vs. standard catheter meta-analysis of trials investigating for less than $\mathbf{1}$ month. 
did not state information about the participants ${ }^{15,18}$. Liedberg ${ }^{25}$ was a three armed trial comparing silver alloy catheters with two non-antiseptic impregnated catheters, one defined in the trial only as a standard catheter and the other a hydrogel catheter (the results for both these standard catheter groups were combined in the meta-analyses). Liedberg ${ }^{26}$ compared the silver alloy catheter with a standard catheter defined as teflonized latex Foley. Verleyen ${ }^{34}$ used a silver alloy hydrogel catheter versus a latex catheter as the comparison standard catheter. Three trials compared silver alloy hydrogel versus a standard silicone catheter ${ }^{19,32,34}$. One trial compared the silver alloy hydrogel catheter versus a standard hydrogel coated catheter ${ }^{15}$. Lundeberg did not define the standard catheter used as a comparison with the silver alloy catheters. Three trials monitored catheter care violations ${ }^{25,34}$.

All the trials defined infection as "bacteriuria >105 CFU/ml." The timing of the outcome measurement varied considerably between trials so results were analyzed as outcomes in two time periods $(<1$ week and $>1$ week). Results were pooled using a fixed effect model and indicated that at $<1$ week of catheterization the risk of asymptomatic bacteriuria (>105 CFU/ml) was statistically significantly reduced in the silver alloy group (RR 0.54, 95\% CI 0.43-0.67, Comparison 01.01.02, Fig. 1). At $>1$ week the estimated effect was smaller but the risk of asymptomatic bacteriuria (>105 CFU/ml) was still less in the silver alloy group (RR 0.64, 95\% CI 0.51-0.80, Comparison 01.01.03, Fig. 1). In summary, there was evidence that the risk of infection was significantly decreased if a patient is catheterized short-term with a catheter coated with silver alloy rather than a standard catheter. For those catheterized for $<1$ week the estimated risk of asymptomatic bacteriuria was at least a half of that with a standard catheter.

The results of a cluster-randomized cross-over trial comparing silver alloy with standard (silicone) catheters $^{35}$ were not included in the meta-analyses because datawere not available prior to crossover (Comparison 01.01.04, Fig. 1). The main outcome measure for this trial was bacteriuria defined as equal or $>105 \mathrm{CFU} / \mathrm{ml}$. Results of the rate of bacteriuria per 1,000 patient days were 2.66 versus 3.35 (RR $0.79,95 \%$ CI $0.63-0.99)$, the rate of bacteriuria per 100 patients was 1.10 versus 1.36 (RR $0.81,95 \%$ CI $0.65-1.01$ ) and the rate of bacteriuria per 100 catheters was 2.13 versus 3.12 (RR 0.68, 95\% CI 0.54-0.86).

\section{Economic outcomes}

Only one trial reported any economic outcomes.35 For the duration of the trial (1 year) silver alloy hydrogel catheters usage resulted in a total estimated catheter-related cost reduction of between $3.3 \%$ and $35.5 \%$. This translated to savings of between US\$14456 and US\$573293.

\section{Adverse effects}

One trial of antiseptic catheters (silver oxide) versus standard catheters included secondary outcomes related to patient comfort and adverse effects of the catheters ${ }^{30}$. They recorded outcome measurements for pain and urethral secretions. No statistically significant difference was found in either outcome but the confidence intervalswere wide: results for reported urethral secretions (RR 0.72, 95\% CI 0.25-2.03); results for patient reporting of pain from the catheters (RR $1.43,95 \%$ CI $0.48-4.27)$.

\section{Antibiotic-impregnated catheter versus standard catheter}

There were two types of antibiotic coated catheters compared with a standard catheter: (i) minocycline and rifampicin combined, or (ii) nitrofurazone. The trials for each were analyzed in two subgroups depending on the type of intervention catheter and grouped into separate outcomes dependent upon duration of catheterization.

\section{Minocycline and rifampicin}

There was only one small trial $(n=124)$ that compared a minocycline and rifampicin impregnated catheter with a standard catheter ${ }^{22}$. This trial included men after radical prostatectomy for prostate cancer and compared a silicone catheter impregnated with minocycline and rifampicin with a standard (silicone) catheter $^{22}$. Outcome measures included bacteriuria $\left(>10^{4} \mathrm{CFU} / \mathrm{ml}\right.$ ) at day 3 , day 7 , and day 14 , and symptomatic UTI (timing not stated) as defined by the health care provider ${ }^{22}$. At $<1$ week, the risk of bacteriuria was about two thirds lower in the antibiotic impregnated catheter group (RR 0.36, 95\% CI 0.18-0.73, Comparison 02.01.01, Fig. 2); however, at $>1$ week the evidence was inconclusive (RR 0.94, 95\% CI 0.86-1.03). One of 56 men in the antibiotic impregnated catheter group had symptomatic bacteriuria compared with 6 of 68 men in the control group (RR 0.20, 95\% CI 0.03-1.63, Fig. 3).

\section{Nitrofurazone}

Four trials compared nitrofurazone-impregnated catheters with standard catheters ${ }^{13,14,16,17}$. Al Habdan used a latex catheter as the standard catheter whilst Lee and Stensballe both used silicone catheters as the 


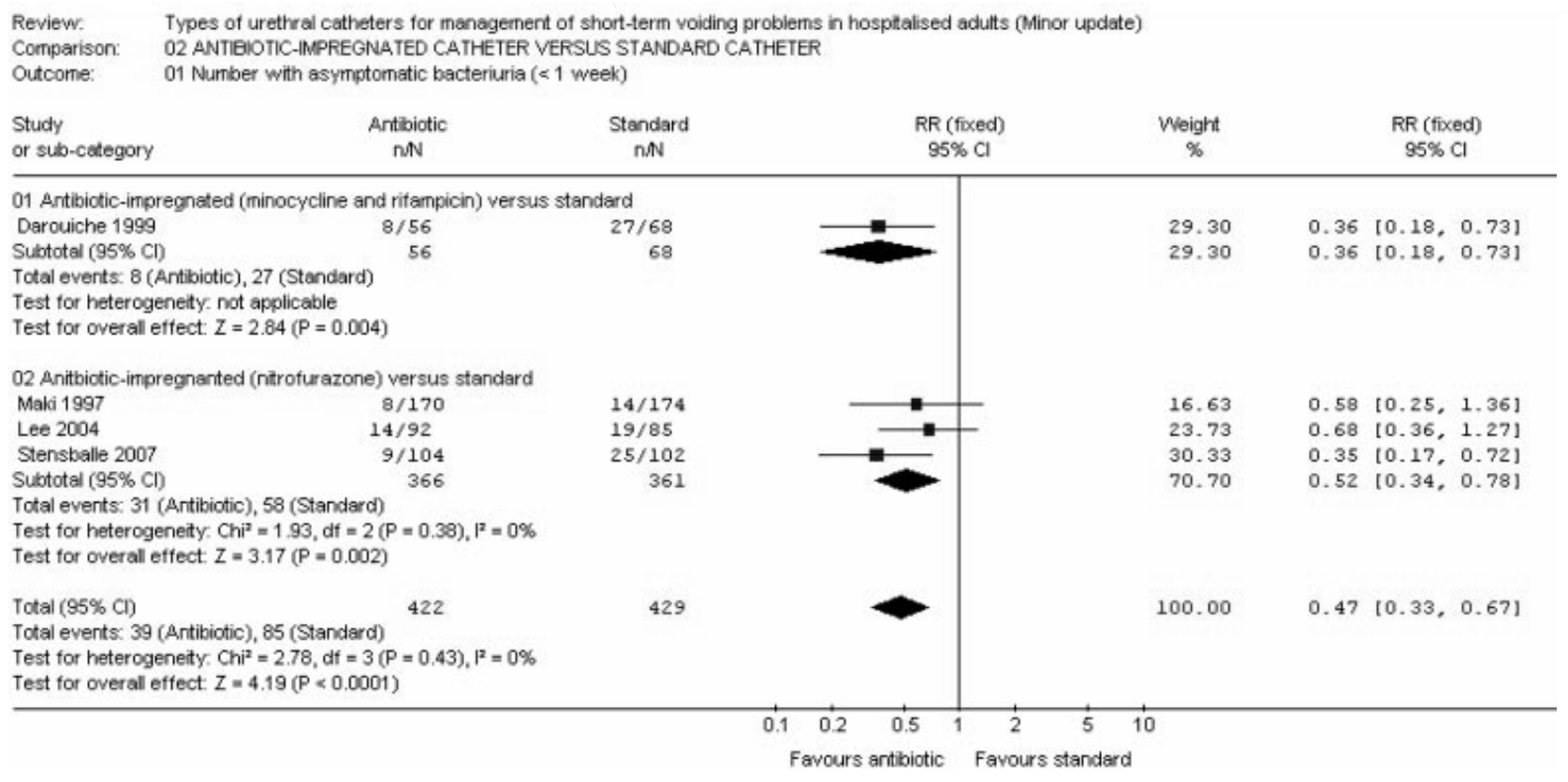

Figure 2. Antibiotic-impregnated catheter versus standard catheter.

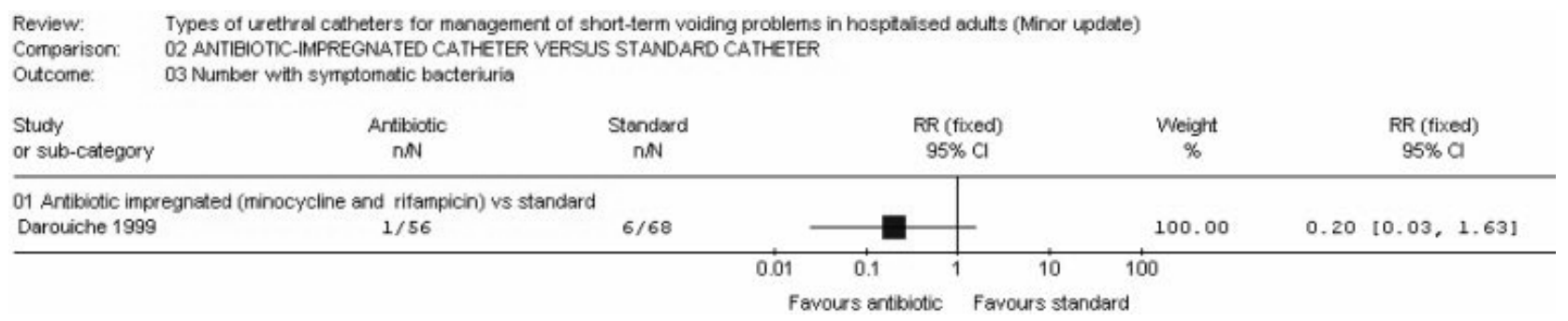

Figure 3. Antibiotic-impregnated catheter vs. standard catheter.

comparison. One study did not report which catheter they used as a comparator ${ }^{16}$. All of the patients in one trial received prophylactic antibiotics pre- and postoperatively ${ }^{13}$, two trials recorded antibiotic use $\mathrm{u}^{16,17}$ whilst two others did not record antibiotic use $\mathrm{u}^{14,15}$.

Only two of the trials adequately described the trial participants. Stensballe and Lee included both men and women. However, Lee included fewerwomen in the treatment group compared to the control (23 vs. 40 ) and more men in the treatment group than the control (69 vs. 45$)$, the reasons for this were not stated in the trial. All outcome measures included bacteriuria (defined as $\geq 10^{3} \mathrm{CFU} / \mathrm{ml}$ ) and, in one trial, associated funguria ${ }^{17}$.

However, the trials differed in their timing of the outcome measurement. Three trials investigated the outcomes at $<1$ week $^{14,16,17}$. Results were pooled using a fixed effect model and indicated that at $<1$ week of catheterization the risk of asymptomatic bacteriuria was statistically significantly reduced in the nitrofurazone impregnated catheter group (RR 0.52, 95\% CI 0.34-0.78, Comparison 02.01.02, Fig. 2). As with minocycline and rifampicin impregnated catheters, at $>1$ week the benefit from nitrofurazone impregnated catheters in preventing bacteriuria was inconclusive, but the numbers were small (RR 0.31, 95\% CI 0.06-1.66, Comparison 02.02.01, Fig. 2).

Interestingly the overall effect for antibiotic impregnated catheters (irrespective of antibiotic type) compared to a standard control was a significant reduction in the risk of asymptomatic bacteriuria at $<1$ week of catheterization (RR 0.47, 95\% CI 0.33-0.67, Comparison 02.01, Fig. 2). However, the two studies which compared antibiotic impregnated catheters to standard control catheters, for more than 1 week, showed no statistically significant difference in the incidence of asymptomatic bacteriuria (RR 0.85 95\% CI 0.76-0.96). Antibiotic-Coated Catheters Versus Antiseptic Catheters No trials were found that addressed this comparison. 
One type of antiseptic catheter versus another type of antiseptic-coated catheter

No trials were found that addressed this comparison.

One type of antibiotic-coated catheter versus another type of antibiotic-coated catheter

No trials were found that addressed this comparison.

\section{One type of standard catheter versus another standard catheter}

Three trials using different outcome measurement compared two types of standard catheters to investigate infection ${ }^{21,28,33}$. The trials were not combined. All three trials compared different types of standard catheters.

The Nickel trial compared silicone with latex catheters using the outcome asymptomatic bacteriuria (defined as $>10^{6} \mathrm{CFU} / \mathrm{ml}$ ), with the final measurement recorded $96 \mathrm{hr}$ post-catheterization. 28 The results of this trial found no evidence of difference in the risk of bacteriuria between the two standard catheters but with wide confidence intervals (RR 1.07, 95\% CI 0.235.01).

The Tidd trial compared three types of standard catheters: hydrophilic polymer-coated latex, uncoated latex and PVC indwelling catheters.33 The outcome of interest was urinary tract infection defined as asymptomatic bacteriuria $103 \mathrm{CFU} / \mathrm{ml}$. The final outcome measurement was recorded at day 5-6 postcatheterization. The evidence from this small trial was insufficient to detect a difference in the risk of infection between any of the three standard catheters compared, with wide confidence intervals: hydron coated latex versus plain latex (RR 0.94, 95\% CI 0.66-1.34); hydron coated latex versus PVC balloon (RR 0.87, 95\% CI 0.631.19); PVC balloon versus plain latex (RR $1.09,95 \% \mathrm{CI}$ $0.81-1.45)$. This trial also had methodologically flawed randomization in some cases.

The Chene trial compared hydrogel with silicone catheters and the outcome measurement was asymptomatic bacteriuria. The results in this trial also found insufficient evidence to say whether or not there was a reduced of risk of infection between the two standard catheters (RR 0.82, 95\% CI 0.46-1.47) ${ }^{21}$.

Three further trials compared different types of standard catheters to investigate urethral side-effects in men ${ }^{24,27,31}$. The outcome measurements differed in all three trials. The Kalambheti trial compared silicone with non-silicone (not defined further) catheters using an outcome measurement of reported burning sensation in the urethra ${ }^{24}$. Results using a fixed effect model found that the risk of a burning sensation in the urethra was less in the silicone catheter group (RR $0.28,95 \%$ CI $0.13-0.60$ ).

The Nacey trial compared silicone with latex catheters and the outcome was urethritis as measured by swabs of urethral discharge ${ }^{27}$. There were fewer cases of urethritis with a silicone catheter (1/50 vs. 11/50; RR 0.09, 95\% CI 0.01-0.68).

Finally, the Talja trial compared three types of standard catheters: hydrogel coated latex, siliconized latex and full silicone.31 The outcome of interest was urethral reaction measured from cytological urethral swab specimens using scanning electron microscopic analysis. Results using a fixed effect model in the comparison of hydrogel coated latex versus siliconized latex indicated no difference in urethral reaction (WMD 0.00, 95\% CI -3.51 to 3.51). Results of the comparison of full silicone versus hydrogel coated latex and siliconized latex found that in both comparisons the risk of urethral reaction was less with a full silicone catheter: full silicone versus hydrogel coated latex (WMD -16.00, 95\% CI -18.84 to 13.16); and full silicone versus siliconized latex (WMD -16.00, 95\% CI -18.96 to -13.04 ).

\section{Discussion}

This systematic review identified 23 eligible trials that addressed three of the six pre-stated comparisons. There were no trials identified that compared one type of antiseptic catheter with another type of antiseptic catheter, or one type of antibiotic catheter with another type of antibiotic catheter. There were also no trials that compared an antiseptic catheter with an antibiotic catheter. Of the included trials, only two reported a secure method of concealment of randomisation ${ }^{17,22}$.

Antiseptic impregnated indwelling urethral catheters versus standard indwelling urethral catheters

This comparison included 12 trials that randomized a total of 3,567 hospitalized adults $(1,782$ catheterizedwith antiseptic catheters and 1,785 with standard catheters) and one crossover trial that randomized hospital wards of 27,878 hospitalized adults. There were two types of antiseptic catheters: silver oxide and silver alloy.

Most of the trials included in the pooled analysis were small with only one trial stating they used a power calculation $^{32}$. The only truly large trial was a cluster- 
randomized cross-over trial ${ }^{35}$. None of the trials reported an adequate method of randomization concealment.

Overall, silver oxide catheters were not found to prevent bacteriuria in short-term catheterized hospitalized adults in the three trials included in the analysis. Subgroup analysis by gender in one trial did suggest that women are less likely to become bacteriuria if they use silver oxide catheters whereas the evidence for men was inconclusive ${ }^{29}$. The same trial also suggested that systemic antibiotic use also decreased the rate of bacteriuria in the silver oxide group, particularly in women. These subgroup analyses should be interpreted cautiously, particularly when the overall result suggests no difference. However, silver oxide catheters are no longer manufactured and therefore these data are no longer clinically relevant.

There was evidence that silver alloy catheters reduced the incidence of asymptomatic bacteriuria. There were limitations to the trials that compared silver alloy hydrogel catheterswith standard catheters. Clinical heterogeneity included diverse populations, use of antibiotics and differences in the standard catheter chosen as the comparison. Only one trial reported catheter care violations ${ }^{26}$. The method of urine specimen collection also differed between the trials, from two trials using the gold standard method of suprapubic puncture ${ }^{34}$, five using various methods to collect the specimen directly from the catheter and one trial collecting the specimen from the drainage bag, which increases the likelihood of bacterial contamination. Therefore, this evidence must be treated cautiously.

Catheterized men and women generally develop UTI in different ways due to their anatomical differences. Men are more likely to develop catheter related UTI via the intraluminal route from a contaminated drainage bag, while in women contamination is more often transurethral when bacteria migrate from the periurethral region after fecal contamination. One major limitation of the silver alloy trials was that subgroup analysis was not possible by gender. Subgrouping of the results by duration of catheterization was possible, however, and this indicated that the protective effect of silver alloy catheters continued from 1 to 2 weeks catheterization.

Only two of the antiseptic catheter trials addressed the secondary objectives of the review. The only trial to investigate patient comfort and adverse effects related to antiseptic catheters was a small silver oxide catheter trial ${ }^{30}$. This inconclusive trial was poorly designed, and the methods for collecting the secondary outcome data were not described.

Overall, from the trials collated in this review, silver oxide catheters were not found to prevent bacteriuria in short term catheterized hospitalized adults. However, silver alloy coated catheters were found to be effective at reducing the incidence of asymptomatic bacteriuria at both less and more than 1 week of catheterization.

\section{Economic evaluation}

There have been no trials that investigate the hypothesis that patients catheterized with antiseptic catheters may develop antimicrobial resistance. One large cluster-randomized cross-over trial completed an economic analysis which found data in favor of silver alloy hydrogel catheters ${ }^{35}$. Silver alloy catheters are significantly more expensive than standard catheters, in fact close to the unit price. The cost estimates derived in the trial used both a low and high approximation of costs and calculated catheter-related cost reduction of between $3.3 \%$ and $35.5 \%$. The limitation of this trial was firstly, the randomization of hospital wards rather than individual patients and secondly, the risk of cross-over of catheters leading to contamination between groups.

Two further studies have been identified that specifically investigated the economic benefits of silver alloy catheters in preventing UTI. $3^{36}$, Plowman developed an illustrative model of the annual costs and benefits associated with the use of silver alloy catheters in hospitalized medical and surgical inpatients in NHS hospitals in England. The model suggested that a reduction in the incidence of UTI of $14.6 \%$ in catheterized medical patients and $11.4 \%$ in catheterized surgical patients would ensure that the cost of silver alloy catheters was the same as standard catheters. Any further reduction in incidence would then result in cost savings. In the second study Saint developed a cost benefit decisionmodel in the USA using a simulated cohort of 1,000 hospitalized general medical, surgical, urologic and intensive care patients requiring short-term catheterization (2-10 days) comparing silver alloy with standard catheters. Results were calculated using a relative risk reduction of bacteriuria with the use of silver alloy catheters of $25 \%$. They calculated that the use of silver alloy catheters could lead to a $47 \%$ relative decrease in the incidence of symptomatic UTI (from 30 to 16 cases per 1,000 patients), with an NNT of 74, as well as a relative decrease in resultant bacteraemia of $44 \%$ (from 4.5 to 2.5 cases), with an NNT of 500. Using amultivariate sensitivity analysis and Monte Carlo simulation, they indicated that silver alloy catheters could provide benefit in all cases and cost savings in $84 \%$ of cases. 
Antibiotic impregnated indwelling urethral catheters versus standard indwelling urethral catheters

This outcome included the results of five trials that randomized a total of 952 hospitalized adults (472 catheterized with antibiotic impregnated catheters and 479 with standard catheters). There were two types of antibiotic impregnated catheters: minocycline combined with rifampicin and nitrofurazone alone.

As with the antiseptic trials, the antibiotic trials included in the pooled analysis were of small numbers of participants. Of the five studies, one of these investigated minocycline and rifampicin impregnated catheters compared to a standard catheter. This relatively small trial was not powered to detect differences in asymptomatic bacteriuria. It included a very limited population (men after radical prostatectomy) and therefore the benefit shown in reducing bacteriuria in those catheterized for less than a week may not be applicable to other groups of adult patients, particularly women. In fact, one of the inclusion criterion was sterile urine prior to catheterization which would be unlikely to be found in more high risk groups. There was not enough evidence to show whether this difference persisted after the first week. Adverse effects, such as antimicrobial resistance to the catheters over time, were not investigated.

There were four other antibiotic trials that investigated nitrofurazone impregnated catheters against standard controls. Of the three trials that investigated the effect of nitrofurazone impregnated catheters against standard catheters at $<1$ week, two of these studies were well designed to minimize bias although small in size $\mathrm{e}^{16,17}$. The other trial was slightly biased in its selection criteria with an unequal distribution of men and women in both the control and treatment groups. Therefore, this must be taken into account when interpreting the results. Overall, nitrofurazoneimpregnated catheters were found to prevent bacteriuria in hospitalized adults who are catheterized for $<1$ week in the three trials included in the analysis.

Although the nitrofurazone impregnated catheters did reduce asymptomatic bacteriuria, the results presented can only be related to catheterization time of $<1$ week. Therewas only one study which investigated catheterization for more than 1 week with nitrofurazone impregnated catheters and these results were not conclusive.

Overall, antibiotic impregnated catheters, regardless of the type of antibiotic, reduce bacteriuria in hospitalized adults who are catheterized for $<1$ week. However, inclusion of more studies would be required to determine if these effects translate to patients who are catheterized for more than 1 week with antibiotic catheters.

One type of standard indwelling urethral catheter versus another type of standard indwelling urethral catheter

This comparison included six trials that randomized a total of 653 hospitalized adults to different types of standard catheters. Three small trials looked at the likelihood of infection between types of standard catheters. There were significant clinical differences between the trials: comparison of different types of standard catheters, inclusion of different types of patients and differing outcome measurements. For these reasons, the data from these trials were not statistically combined. None of the trials, however, provided sufficient evidence to suggest whether or not any of the standard catheters results in a decreased rate of bacteriuria compared to another standard catheter.

Another three trials investigated adverse effects of standard catheters, in particular urethral side effects in men. Again the trials were clinically heterogenous. All the trials included different outcome measurement and the catheters compared were diverse. The results indicated that siliconized catheters were less likely to result in adverse urethral effects inmen, but each outcome was addressed only in single small trials.

\section{Conclusions}

Implications for practice

1. Are antiseptic impregnated indwelling urethral catheters better than standard indwelling urethral catheters?

The evidence suggests that silver alloy catheters prevent asymptomatic bacteriuria in the short-term catheterized patient, although the trials are generally of poor quality.

2. Are antibiotic impregnated indwelling urethral catheters better than standard indwelling urethral catheters?

The evidence suggests that antibiotic impregnated catheters reduce asymptomatic bacteriuria in hospitalized adults catheterized short-term, although there were only four trials included in the analysis. The current evidence suggests that this is only true up to 1 week of catheterization as there as no significant effect in those patients catheterized for more than 1 week. 
3. Are antibiotic impregnated indwelling urethral catheters better than antiseptic impregnated indwelling urethral catheters?

None of the trials included in the review address this question.

4. Is one type of antiseptic impregnated indwelling urethral catheter better than another type of antiseptic impregnated indwelling urethral catheter?

None of the trials included in the review address this question.

5. Is one type of antibiotic impregnated indwelling urethral catheter better than another type of antibiotic impregnated indwelling urethral catheter?

None of the trials included in the review address this question.

6. Is one type of standard indwelling urethral catheter better than another type of standard indwelling urethral catheter?

No standard catheter was found to be better than another in terms of reducing the risk of bacteriuria in hospitalized adults catheterized short-term. Siliconized catheters may be less likely to cause urethral side effects in men. However, these results should be interpreted with some caution as the trials were small and the outcome definitions and specific catheters compared differed.

\section{Implications for research}

While silver alloy and antibiotic impregnated catheters appear to be more effective than standard catheters in reducing the likelihood of bacteriuria in hospitalized adults catheterized short-term, the evidence for this comes from trials which were small and of poor to moderate quality. Larger, well powered, better quality randomized trials with adequate allocation concealment and blinding of outcome assessors are required. Trial reports also need to follow the CONSORT statement: within this review only one trial reports according to the CONSORT statement.

Four high priority issues for future research to determine the effect of type of indwelling urethral catheter on the risk of urinary tract infection are:

1. The choice of outcome measurements (e.g., symptomatic UTI, bacteraemia).

2. Identification of at risk sub-groups (e.g., women, immunocompromised patients).
3. The effect of co-intervention with systemic antibiotics.

4. Consideration of possible adverse effects, such as allergies, resistance, etc.

Trials should be conducted to compare both antibiotic impregnated catheters and silver alloy or other antiseptic catheters with standard catheters.

\section{Potential conflict of interest}

The two authors who updated this review (KS \&TL) are both currently involved in a large, multicenter, RCT that aims to investigate types of urethral catheter for reducing symptomatic urinary tract infections in hospitalized adults requiring short-term catheterization. This trial will investigate antibiotic (nitrofurazone) impregnated and antiseptic (silver alloy) coated catheters against standard polytetraflouroethylene (PTFE) catheters. The trial aims to disseminate its findings by 2010 .

\section{Acknowledgments}

We are grateful to Professor James N'Dowand the Academic Urology Unit, University of Aberdeen, for support and encouragement. Also, the previous authors of this review, Jane Brosnahan, Andrew Jull, and Catherine Tracy. This review is an update of one originally published online on January 26, 2004, Issue 1 of the Cochrane Library.

\section{References}

1. Nosocomial Infection National Surveillance Service. Surveillance of hospitalacquired bacteraemia in English Hospitals. London: Public Health Laboratory Service, 2000.

2. Plowman R, Graves N, Griffin M, et al. The socio-economic burden of hospital acquired bloodstream infection. London: Public Health Laboratory, 1999.

3. Plowman R, Graves N, Esquive J, et al. An economic model to assess the cost and benefits of the routine use of silver alloy coated urinary catheters to reduce the risk of urinary tract infections in catheterized patients. J Hosp Infect 2001; 48: $38-42$.

4. Stamm WE. Urinary tract infection. In: Bennett JV, Brachmand PS, editors. Hospital infections. 4th edition. Philadelphia: Lippincott-Raven; 1998. pp 477-85.

5. Maki DG, Tambyah PA. Engineering out the risk for infection with urinary catheters. Emerg Infect Dis 2001; 7: 342-7.

6. Jarvis RJ, Martone WJ. Predominant pathogens in hospital infections. Br Soc Antimicrob Chemother 1992; 29: 19-24.

7. Campbell JB, Moore KN, Voaklander DC, et al. Complications associated with clean intermittent catheterization in children with spina bifida. J Urol 2004; 171: $2420-2$. 
8. Pomfret I. Catheter care in the community. Nurs Stand 2000; 14: 46-53.

9. Robinson J. Urethral catheter selection. Nurs Stand 2001;15:39-42.

10. Saint S, Elmore JG, Sullivan SD, et al. The efficacy of silver alloy-coated urinary catheters in peventing urinary tract infection: A meta-analysis. Am J Med 1998; 105: 236-41.

11. Saint S. Prevention of nosocomial urinary tract infections. In: Markowitz AJ, editor. Making health care safer: A critical analysis of patient safety practices, Vol. 43. San Francisco: Agency for Healthcare Research and Quality; 2001: pp 149-55.

12. Schumm K, Lam TBL. Types of urethral catheters for management of shortterm voiding problems in hospitalised adults. Cochrane Database Syst Rev 2008; Art. No.: CD004013.10.1002/14651858.CD004013.pub3. References to studies.

13. Al Habdan I, Sadat-Ali M, Corea JR, et al. Assessment of nosocomial urinary tract infections in orthopaedic patients: A prospective and comparative study using two different catheters. Int Surg 2003; 88: 152-4.

14. Lee SJ, Kim SW, Cho YH, et al. A comparative multicentre study on the incidence of catheter-associated urinary tract infection between nitrofurazone-coated and silicone catheters. Int J Antimicrob Agents 2004; 24: S65-9.

15. Liedberg H, Lundeberg T. Prospective study of incidence of urinary tract infection in patients catheterized with Bard hydrogel and silver-coated catheters or Bard hydrogel-coated catheters (Abstract number 769). J Urol 1993; 149: 405A.

16. Maki DG, Holcomb RG. A report on the randomized, controlled clinical trial of the nitrofurazone-impregnated, antibacterial, indwelling urinary catheter. Stewartville, Minnesota: Rochester Medical Corporation; 1997.

17. Stensballe J, Tvede M, Looms D, et al. Infection risk with nitrofurazoneimpregnated urinary catheters in trauma patients: A randomized trial. Ann Intern Med 2007; 147: 285-93.

18. Lundeberg T. Prevention of catheter-associated urinary tract infections by use of silver impregnated catheters. Lancet 1986; 2: 1031.

19. Maki DG, Knasinski V, Halvorson K, et al. A novel silverhydrogelimpregnated indwelling urinary catheter reduces CAUTIs: A prospective double-blind trial. Infect Control Hosp Epidemiol 1998; 1992: 682.

20. Niel-Weise BS, Arend SM, van den Broek PJ. Is there evidence for recommending silver-coated urinary catheters in guidelines? J Hosp Infect 2002; 52: 81-7.

21. Chene G, Boulard G, Gachie JP. Essai controle d'un nouveau materiau pour enduire les sondes urnaires. Agressologie 1990; 31: 499-501.
22. Darouiche RO, Smith JA, Hanna H, et al. Efficacy of antimicrobialimpregnated bladder catheters in reducing catheter-associated bacteriuria: A prospective, randomised, muticentre clinical trial. Urology 1999; 54: 976-87.

23. Johnson JR, Roberts PL, Olsen RJ, et al. Prevention of catheter-associated urinary tract infection with a silver oxidecoated urinary catheter: Clinical and microbiologic correlates. J Infect Dis 1990; 162: 1145-50.

24. Kalambheti K. Siliconized Foley catheters. Am J Surg 1965; 110: 935-6.

25. Liedberg T, Lundeberg PE. Refinements in the coating of urethral catheters reduces the incidence of catheterassociated bacteriuria. Eur Urol 1990; 17: 236-40.

26. Liedberg $\mathrm{H}$, Lundeberg $\mathrm{T}$. Silver alloy coated catheters reduce catheterassociated bacteriuria. Br J Urol 1990; 65: 379-81.

27. Nacey JN, Tulloch AGS, Ferguson AS. Catheter-induced urethritis: A comparison between latex and silicone catheters in a prospective clinical trial. Br J Urol 1984; 57: 325-8.

28. Nickel JC, Feero P, CostertonW, et al. Incidence and importance of bacteriuria in postoperative, short-term urinary catheterization. Can J Surg 1989; 32: 131-2.

29. Riley DK, Classen DC, Stevens LE, et al. A large randomized clinical trial of a silver-impregnated urinary catheter: Lack of efficacy and staphyloccal superinfection. Am J Med 1995; 98: 349-56.

30. Takeuchi H, Hida S, Yoshida O, et al. Clinical study on efficacy of a Foley catheter coated with silver-protein in prevention of urinary tract infections. Hinyokika Kiyo 1993; 39: $293-8$.

31. Talja M, Korpela A, Jarvi K. Comparison of urethral reaction to full silicone hydrogel-coated and siliconised latex catheters. Br J Urol 1990; 66: 652-7.

32. Thibon P, Le Coutour X, Leroyer R, et al. Randomized multicentre trial of the effects of a catheter coated with hydrogel and silver salts on the incidence of hospital-acquired urinary tract infection. Hosp Infect 2000; 45: 117-24.

33. Tidd MJ, Gow JG, Pennington JH, et al. Comparison of hydrophilic polymercoated latex, uncoated latex and PVC indwelling balloon catheters in the prevention of urinary infection. Br J Urol 1976; 48: 285-91.

34. Verleyen P, De Ridder D, Van Poppel H, et al. Clinical application of the Bardex IC Foley catheter. Eur Urol 1999; 36: $240-6$.

35. Karchmer TB, Giannetta ET, Muto CA, et al. A randomized crossover study of silver-coated urinary catheters in hospitalized patients. Arch Intern Med 2000; 160: 3294-8.

36. SaintS, Veenstra DL, SullivanSD, et al. The potential clinical and economic benefits of silver alloy urinary catheters in preventing urinary tract infection. Arch Intern Med 2000: 2000; 160: 2670-5. 